\title{
Evaluation of a patient-specific algorithm for predicting distribution for convection-enhanced drug delivery into the brainstem of patients with diffuse intrinsic pontine glioma
}

\author{
Eva Wembacher-Schroeder, BS, ${ }^{1}$ Nicole Kerstein, MSc, ${ }^{1}$ Evan D. Bander, MD, ${ }^{2,3}$ \\ Neeta Pandit-Taskar, MD, ${ }^{4}$ Rowena Thomson, PhD, ${ }^{1}$ and Mark M. Souweidane, MD ${ }^{2,3}$ \\ ${ }^{1}$ Brainlab AG, Munich, Germany; 2Department of Neurological Surgery, Weill Medical College of Cornell University, New York, \\ New York; and Departments of ${ }^{3}$ Neurological Surgery and ${ }^{4}$ Radiology, Memorial Sloan Kettering Cancer Center, New York, New \\ York
}

\begin{abstract}
OBJECTIVE With increasing use of convection-enhanced delivery (CED) of drugs, the need for software that can predict infusion distribution has grown. In the context of a phase I clinical trial for pediatric diffuse intrinsic pontine glioma (DIPG), CED was used to administer an anti-B7H3 radiolabeled monoclonal antibody, iodine-124-labeled omburtamab. In this study, the authors retrospectively evaluated a software algorithm (iPlan Flow) for the estimation of infusate distribution based on the planned catheter trajectory, infusion parameters, and patient-specific MRI. The actual infusate distribution, as determined on MRI and PET imaging, was compared to the distribution estimated by the software algorithm. Similarity metrics were used to quantify the agreement between predicted and actual distributions.

METHODS Ten pediatric patients treated at the same dose level in the NCT01502917 trial conducted at Memorial Sloan Kettering Cancer Center were considered for this retrospective analysis. T2-weighted MRI in combination with PET imaging was used to determine the distribution of infusate in this study. The software algorithm was applied for the generation of estimated fluid distribution maps. Similarity measures included object volumes, intersection volume, union volume, Dice coefficient, volume difference, and the center and average surface distances. Acceptable similarity was defined as a simulated distribution volume (Vd Sim) object that had a Dice coefficient higher than or equal to 0.7 , a falsenegative rate (FNR) lower than 50\%, and a positive predictive value (PPV) higher than $50 \%$ compared to the actual Vd (Vd PET).
\end{abstract}

RESULTS Data for 10 patients with a mean infusion volume of $4.29 \mathrm{ml}$ (range $3.84-4.48 \mathrm{ml}$ ) were available for software evaluation. The mean Vd Sim found to be covered by the actual PET distribution (PPV) was $77 \% \pm 8 \%$. The mean percentage of PET volume found to be outside the simulated volume (FNR) was $34 \% \pm 10 \%$. The mean Dice coefficient was $0.7 \pm 0.05$. In 8 out of 10 patients, the simulation algorithm fulfilled the combined acceptance criteria for similarity.

CONCLUSIONS iPlan Flow software can be useful to support planning of trajectories that produce intraparenchymal convection. The simulation algorithm is able to model the likely infusate distribution for a CED treatment in DIPG patients. The combination of trajectory planning guidelines and infusion simulation in the software can be used prospectively to optimize personalized CED treatment.

https://thejns.org/doi/abs/10.3171/2020.11.PEDS20571

KEYWORDS convection-enhanced delivery; MRI; surgical planning; diffuse intrinsic pontine glioma; brainstem; oncology

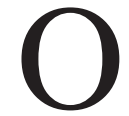
NE major obstacle in the treatment of CNS tumors is efficient drug delivery to the intended target tissue. Systemic delivery such as intravenous injection or oral administration leads to poor transit of drugs across the blood-brain barrier (BBB). ${ }^{1}$ Dose escalation of systemically administered antineoplastics, a common tactic to enhance target tissue availability, is limited by systemic toxicity and adverse side effects. Convection-enhanced delivery (CED) is an alternative delivery method that reduces the systemic exposure to drugs by directly in-

ABBREVIATIONS AD = average surface distance; $C D=$ center distance; $C E D=$ convection-enhanced delivery; $D I P G=$ diffuse intrinsic pontine glioma; FN = false negative; FNR = FN rate; FP = false positive; MSKCC = Memorial Sloan Kettering Cancer Center; PPV = positive predictive value; TP = true positive; Vd = distribution volume; Vd PET = Vd of 124l-omburtamab determined for PET images; Vd Sim = simulated Vd; 124l-omburtamab = iodine-124-labeled omburtamab.

SUBMITTED June 30, 2020. ACCEPTED November 19, 2020.

INCLUDE WHEN CITING Published online May 14, 2021; DOI: 10.3171/2020.11.PEDS20571. 
fusing the therapeutic agent to the desired site. ${ }^{2}$ CED has been used safely in clinical trials for several years for the treatment of brain tumors in children and adults. ${ }^{3,4}$ While CED has been repeatedly shown to be safe, however, most clinical trials of drugs for malignant primary brain tumors have not reproducibly demonstrated clinical benefit. ${ }^{5} \mathrm{CED}$ allows for homogenous concentration throughout the distribution volume (Vd); the shape of the infusion is influenced by infusion parameters and patient-specific factors that make this treatment unpredictable. ${ }^{6}$ iPlan Flow is an FDA-cleared and CE-marked software that enables clinicians to optimize direct infusions of a therapeutic agent. The software uses MRI to allow for patient-specific planning of catheter positions and to perform prospective simulations of drug distribution to determine the expected target coverage based on a preplanned catheter setup, infusion parameters, and individual tissue properties. The algorithm has been previously described in detail and was initially validated for supratentorial peritumoral applications by comparison of the distribution of iodine-123-labeled human serum albumin (123I-HSA) in patients diagnosed with glioblastoma multiforme (GBM) with the predicted infusate distribution..$^{7-9}$ Later, the software was adapted and revalidated in 20 infusions of gadolinium into the thalamus and the putamen of nonhuman primates. ${ }^{10}$

The purpose of this study was to evaluate this algorithm for brainstem applications by using data of radiolabeled infusions during the treatment of diffuse intrinsic pontine glioma (DIPG). PET and MRI data were collected as part of a phase I study conducted at Memorial Sloan Kettering Cancer Center (MSKCC) using a single dose of iodine-124-labeled omburtamab (124I-omburtamab; Y-mAbs Therapeutics) delivered by CED into the brainstem of children with DIPG. Omburtamab is a murine antibody that binds to the antigen B7-H3, a transmembrane protein known to be overexpressed in high-grade solid tumors, including DIPG. ${ }^{11,12}$ Omburtamab is conjugated to iodine-124, a radioisotope that has positron emission allowing for PET imaging. In addition, the positron energy of $511 \mathrm{keV}$ can deliver a radiation dose to the tissues, which can be therapeutic. Thus, 124I-omburtamab incorporates a therapeutic agent with an imaging moiety into a single theranostic compound. The Vd of 124I-omburtamab determined for PET images (Vd PET) was compared to the distribution estimated by the simulation algorithm (Vd Sim). Similarity metrics were used to quantify the similarity between the actual and the estimated infusate distribution.

\section{Methods}

\section{Patients and Treatment}

Patients in this study were enrolled in a dose-escalation, phase I, single-center trial in which CED was used to deliver a radiolabeled monoclonal antibody (124I-omburtamab) into the brainstem of DIPG patients. ${ }^{13}$ This trial was registered at https://clinicaltrials.gov (NCT01502917) and was approved by the MSKCC Institutional Review Board. Informed consent was obtained from all patients/ legal guardians. Dose-escalation cohorts were planned based on standard $3+3$ rules, with 39 patients enrolled between 2012 and 2020. Patients were treated with a single infusion 4-14 weeks after standard conventional external-beam radiation therapy (5940 cGy). For the study described herein, analysis was confined to dose level 7 , which included 15 patients receiving the same dose prescription, but with stepwise increases in infusion rate. This dose level allowed for analysis of a homogeneous cohort at a clinically relevant infusion volume. Five patients had to be excluded from analysis due to lack of required MRI studies $(n=1)$ and not meeting the defined inclusion criteria with respect to catheter tip location $(n=4)$, described in more detail below (Fig. 1). A total of 10 patients participated in the remaining analysis.

\section{Catheter Placement}

MRI-guided stereotactic placement of the infusion catheter (Brainlab Flexible Catheter) was performed under general anesthesia using an MRI-compatible system (ClearPoint Neuro, Inc.). ${ }^{14,15}$ The insertion via a supratentorial route was performed using real-time intraoperative MR guidance with a 1.5-T Siemens scanner. A bone anchor was used to secure the catheter in place and the guidance device was removed. The infusion of 124I-omburtamab via CED was administered outside the MR environment with the child awake in a monitored setting over a period of several hours.

\section{MR Imaging}

MRI included pregadolinium isotropic 3D T1-weighted gradient echo (GRE) (slice thickness $1 \mathrm{~mm}$, matrix $256 \times$ 256, slice spacing $0.5 \mathrm{~mm}$ ), 2D axial T2-weighted (slice thickness $3 \mathrm{~mm}$, matrix $512 \times 256$, slice spacing $3 \mathrm{~mm}$ ), 2D axial T2-weighted FLAIR (slice thickness $3 \mathrm{~mm}$, matrix $320 \times 224$, slice spacing $3 \mathrm{~mm}$ ), 3D axial SWAN (slice thickness $1 \mathrm{~mm}$, matrix $320 \times 224$, slice spacing $0.5 \mathrm{~mm}$ ), postgadolinium 3D T1-weighted GRE (slice thickness 1 $\mathrm{mm}$, matrix $256 \times 256$, slice spacing $0.5 \mathrm{~mm}$ ), and postgadolinium 2D axial T1-weighted FLAIR (slice thickness $3 \mathrm{~mm}$, matrix $288 \times 192$, slice spacing $3 \mathrm{~mm}$ ) sequences. Diffusion tensor images provided input data for the simulation algorithm and were acquired using an echo-planar imaging (EPI) sequence (35 directions, $128 \times 128$ matrix [zipped $\times 2$ ]) of $2 \mathrm{~mm}$, with B values of 0 and $1000 \mathrm{~mm}^{2} /$ sec. MRI was performed within 14 days prior to surgery, intraoperatively, and immediately after removal of the infusion catheter.

\section{PET/CT Imaging}

Imaging was performed at multiple time points using a GE PET/CT scanner. PET/CT scans of the head followed by body imaging were performed within 1-6 hours, and then at $48 \pm 24$ hours, $96 \pm 24$ hours, and $7 \pm 1$ days after completion of infusion. Images were acquired in a 3D format with 5 minutes per field of view.

\section{Vd After CED of 124I-Omburtamab}

124I-omburtamab PET/CT imaging was used for imaging of antibody distribution. Distribution assessment generally involves using a thresholding method. However, there is no validated standardized method for setting 


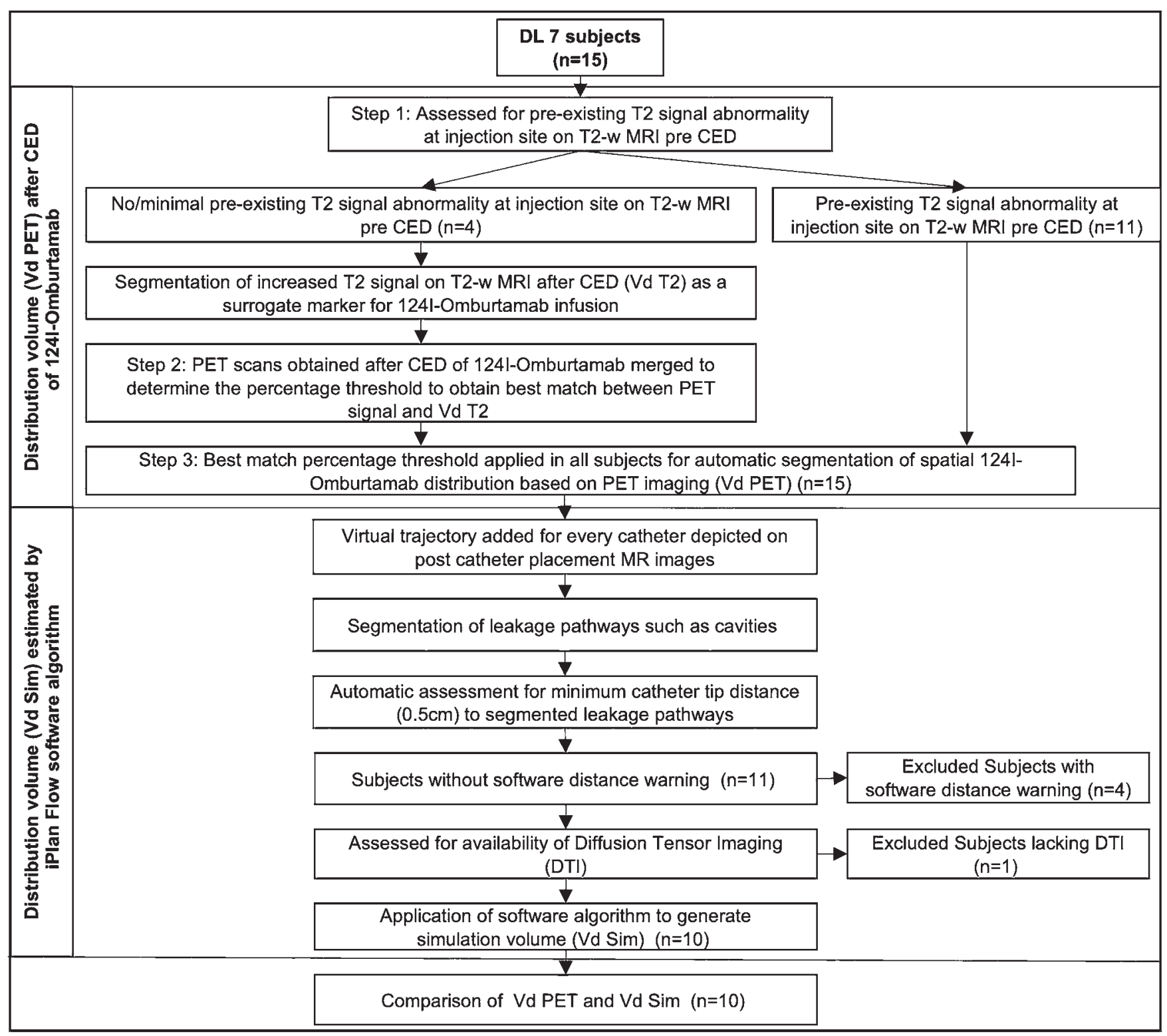

FIG. 1. Patient inclusion criteria and subanalysis flowchart. $\mathrm{T} 2-\mathrm{w}=\mathrm{T} 2$-weighted.

a threshold when segmenting spatial distribution on PET imaging, especially for CED distribution of a radiolabeled antibody ${ }^{16,17}$ Previous studies have shown that T2-weighted MRI allows estimation of the volume of infusate distribution after CED for patients with only minimal preexisting T2 signal abnormality at the injection site. ${ }^{18,19}$ Therefore, to overcome these limitations, T2-weighted MRI in combination with PET imaging was used to determine the location of infusate distribution (Fig. 1).

The following steps were applied.

\section{Step 1-Definition of Vd Based on T2-Weighted MRI}

Minimal or no preexisting T2 signal abnormality at the injection site in 4 patients allowed for reliable delineation of T2 signal change immediately after CED. A thresholdbased segmentation feature incorporated in iPlan Flow was used to generate the infusate distribution volumes, referred to as Vd T2. Figure 2 shows an example for the increased T2 signal intensity after CED. Image processing for all 4 patients was first performed by operator 1 , a qualified radiological technologist with over 10 years of experience in using iPlan Flow. Two additional operators, independently and blinded to the results of operator 1 , completed segmentation for the 4 patients. Operator 2 was experienced in using the software, whereas operator 3 had little experience with the method and the software. Operator 3 reevaluated all 4 infusions in a second run. Interoperator variability was estimated by calculating the standard deviation, the variance, and the Dice coefficients.

Step 2-Definition of PET Threshold to Match Vd Based on T2

For the 4 patients with minimal or no preexisting T2 


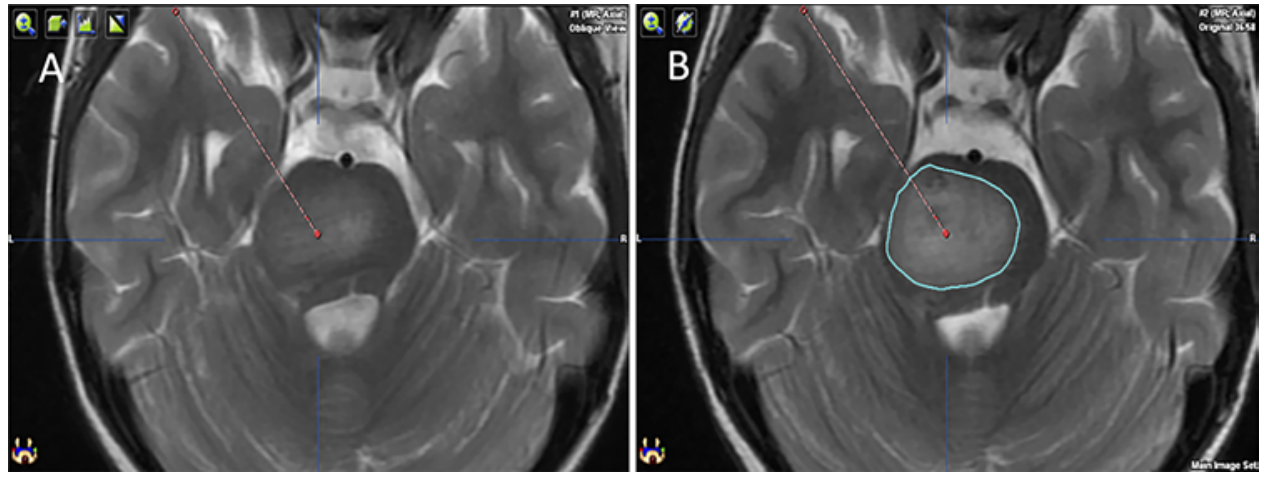

FIG. 2. Screenshot showing the virtual trajectory (red dashed lines) overlaid on an axial view 2D T2-weighted MR image. A: Baseline scan shows minor preexisting T2 signal abnormality at the injection site. B: Postinfusion scan shows outline (turquoise-outlined area) of visually increased signal area after injection of 124 l-omburtamab.

signal abnormality at the injection site, operator 3 imported PET/CT scans obtained within 1-6 hours of the conclusion of the 124I-omburtamab infusion into iPlan Flow software to determine the percentage threshold required to obtain the best match between PET and Vd T2 volumes. The PET signal for 124I-omburtamab infusion was displayed as uptake in becquerel per milliliter. An automatic segmentation method was used to generate objects at $10 \%$, $20 \%, 30 \%, 40 \%, 50 \%$, and $60 \%$ of the maximum uptake value. The threshold value of $30 \%$ was found to be most useful when looking at volume differences from the Vd T2 volume. Intervals were then incrementally adjusted around that threshold value to determine the best match threshold for each of the 4 patients. Finally, the mean of the best match threshold values was calculated and applied in the 4 patients again. The resulting objects were then compared with respect to their geometrical and volumetric differences from the Vd T2 volume. Metrics used for comparison are described in the Comparison of Objects section.

\section{Step 3-Segmentation of Spatial 124I-Omburtamab Distribution}

The threshold determined in step 2 was then applied by operator 3 in all patients for automatic segmentation of the spatial 124I-omburtamab distribution, referred to as Vd PET.

\section{Generation of Simulation Volumes}

Operator 1, who was blinded to the results of PET imaging, analyzed the intraprocedural MRI scans to identify the actual catheter position on MRI and added a digital trajectory. Before simulating the infusate distribution, cavities were segmented based on 3D T1-weighted MRI. These fluid-filled structures are prone to cause pooling and/or leakage of the infusate and therefore prevent intraparenchymal convection. ${ }^{5}$ The segmentation results were verified by medical experts (M.M.S. and E.D.B.). A minimum distance of $0.5 \mathrm{~cm}$ from the catheter tip to potential leakage pathways is recommended in the literature and was defined as an inclusion criterion in this project. ${ }^{20} \mathrm{iPlan}$ Flow allows for geometrical visualization of this minimal distance and provides a warning for catheters not meeting this criterion (Fig. 3). As the simulation result is valid for trajectories within parenchyma, with sufficient distance from the catheter tip to potential leakage/pooling pathways, patients with an active warning were excluded from further analysis $(n=4)$. MR images for the remaining 11 patients were then passed to operator 3 for generation of the simulation volumes using the algorithm previously described by Rosenbluth et al. ${ }^{10}$

The algorithm uses information derived from diffusion tensor imaging (DTI) to estimate tissue properties such as pore fraction and hydraulic conductivity. ${ }^{10}$ DTI was lacking for 1 patient, which resulted in a total of 10 patients with all data available for further analysis. The outer diameter of the catheter used in this study increased in two steps from $0.67 \mathrm{~mm}$ at the proximal tip to its widest outer diameter of $2.11 \mathrm{~mm}$ at the distal catheter shaft. An outer diameter of $1 \mathrm{~mm}$ was chosen in the software settings to most closely match the catheter dimensions at the proximal stepped tip. A stepped flow rate plan with 10-minute intervals was used until the maximum flow rate of $10 \mu \mathrm{l} /$ min was reached. In the simulation software, an average overall flow rate calculated from the duration of infusion and the total infusion volume was used for each patient. The resulting infusate distribution estimation was referred to as Vd Sim.

\section{Comparison of Objects}

ObjectComparer, a software developed by Brainlab for the validation of medical segmentation, was used to compare the objects of interest to their reference objects. The following parameters were used for analysis: object volumes, intersection volume, union volume, Dice coefficient, volume difference, and center and average surface distance. The Dice coefficient was calculated to measure the similarity of the objects of interest to their reference objects. The Dice coefficient ranges between 0 and 1 , where 0 indicates no overlap and 1 indicates exact overlap. ${ }^{21} \mathrm{~A}$ value of 0.7 is defined as clinically acceptable ${ }^{22} \mathrm{ac}-$ cording to this equation, for which TP is true positive, FP is false positive, and FN is false negative: Dice coefficient $=(2 \times \mathrm{TP}) /[(2 \times \mathrm{TP})+\mathrm{FP}+\mathrm{FN}]$.

Overlap-based metrics defined as TPs, FPs, and FNs (Fig. 4A) were used for comparison of $\mathrm{Vd}$ Sim to $\mathrm{Vd}$ PET. ${ }^{23}$ Positive predictive value (PPV) was defined as the percentage of Vd Sim that was found to be covered by Vd 

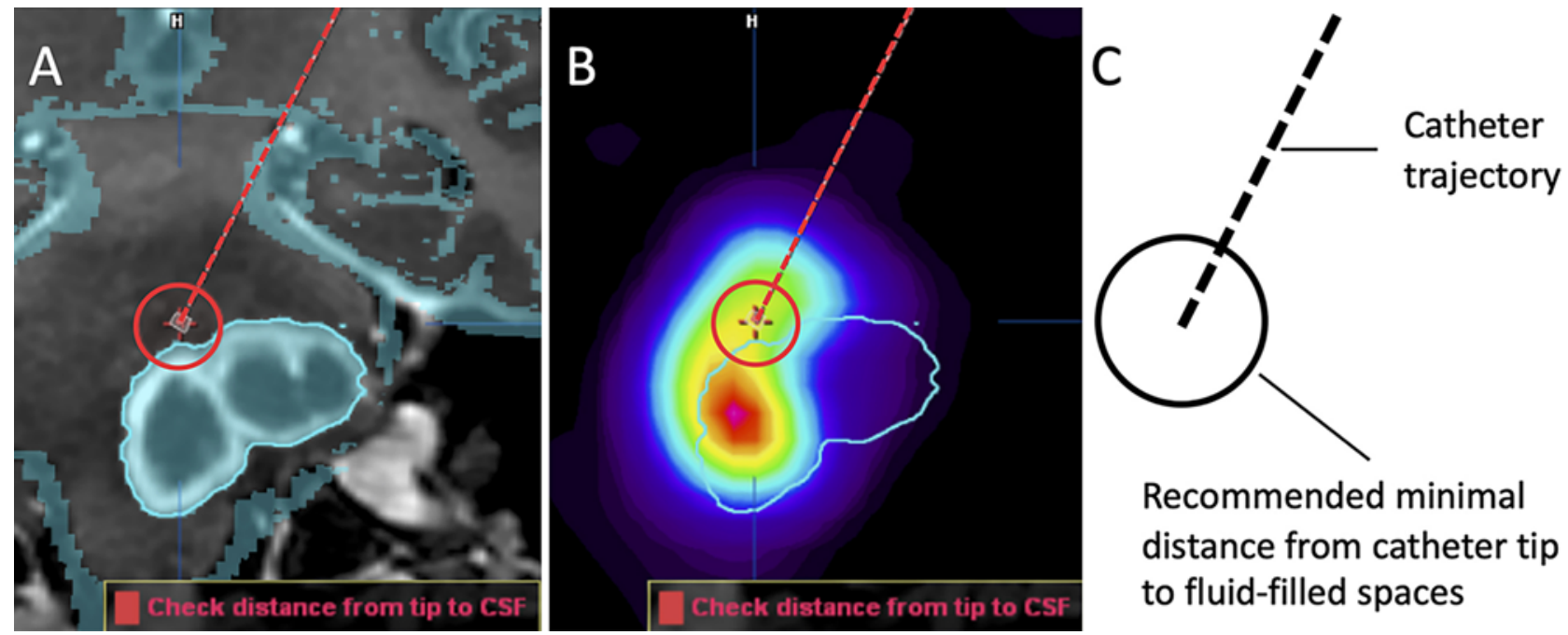

FIG. 3. Screenshot of the images from a patient whose data were excluded from further analysis due to a software distance warning that the catheter tip was planned too close to the potential leakage pathway. A: Image showing the autosegmented fluid-filled areas (turquoise), as well as a virtual catheter trajectory (red dashed line) and the guideline (red circle at virtual catheter tip) of the recommended minimal distance of $0.5 \mathrm{~cm}$ from the catheter tip to the fluid-filled spaces overlaid on a coronal view 3D T1-weighted MR image. A warning text box ("Check distance from tip to CSF") actively notifies the user that the catheter tip is planned too close to the potential leakage pathway. B: The actual infusion derived from this catheter is shown on the PET image. The focal increased PET activity corresponds with pooling of the infusate within the cystic compartment of the outlined area. C: Schematic of the catheter tip to the CSF distance guideline.

PET. The FN rate (FNR) was defined as the percentage of Vd PET that was outside Vd Sim.

The acceptance criteria were met if the comparison of Vd PET objects to the Vd Sim objects resulted in a Dice coefficient greater than or equal to 0.7 , if the $\mathrm{FN}$ rate was lower than 50\%, and if the mean PPV was higher than $50 \% .10$

The following distance metrics were not part of the acceptance criteria, but a smaller value suggests a lower distance and therefore greater similarity between the compared objects (Fig. 4B). The average surface distance (AD) is the average of all distances between the surfaces of the two objects $\mathrm{A}$ and $\mathrm{B}$ and is defined as $\mathrm{AD}(\mathrm{A}, \mathrm{B})=$ $\max [\mathrm{d}(\mathrm{A}, \mathrm{B}), \mathrm{d}(\mathrm{B}, \mathrm{A})]$, with

$$
d(A, B)=\frac{1}{N} \sum_{a \in A} \min \|a-b\| \text { with }[b \in B] .
$$

The center distance (CD) describes the Euclidean distance between the two centers of mass of two objects. Each center of mass is calculated using the volume of the individual object.

\section{Results \\ Patient Demographics}

Ten children (6 female, 4 male; age range 3-11 years, mean $7.0 \pm 2.3$ years) were treated at the same dose level, dose level 7, as in the NCT01502917 study and completed analysis in this subgroup study. The mean infusion volume in the 10 patients was $4.29 \mathrm{ml}$ (range $3.84-4.48 \mathrm{ml}$ ), infused over several hours (mean 710 minutes, range 469918 minutes). This dose level within the trial was chosen for analysis in order to maintain uniformity in treatment parameters (prescribed dose of $4 \mathrm{mCi}$, maximal flow rate of $10 \mu \mathrm{l} / \mathrm{min}$ ) and a clinically relevant infusion volume.

\section{Determination of Vd After CED of 124l-Omburtamab}

In order to define a threshold for Vd PET, 4 patients with minimal pre-CED T2 signal were used to identify the threshold that best matched Vd PET and Vd T2. Delineation of the T2 signals induced by CED in these 4 patients was robust. Interoperator variability showed an SD in volume of $1.3 \mathrm{~cm}^{3}$ for an average T2 volume of $11.5 \mathrm{~cm}^{3}$ and a variance of 1.1 between the three operators. Dice coefficients ranging from 0.7 to 0.9 were measured between the outcomes of operators 1 and 3 .

Vd T2 objects segmented by operator 1 were used as reference objects for quantitative comparison with objects
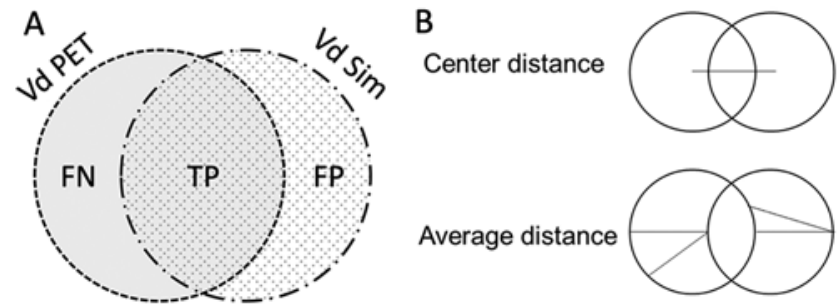

FIG. 4. A: Visualization of metrics used to compare simulated volume (Vd Sim) to distribution volume as determined based on PET imaging ( $V d$ PET). The TP region was defined as the intersecting volume between Vd Sim and Vd PET. The FN volume was described by Vd PET minus the intersecting volume (TP). The FP was defined by Vd Sim minus the intersecting volume (TP). B: Visualization of the theory behind the $C D$ and $A D$. 
TABLE 1. Definition of PET threshold for segmentation of the distribution of 124l-omburtamab, based on PET signal, and comparison between Vd PET at $27 \%$ and operator 1 reference Vd T2 object

\begin{tabular}{|c|c|c|c|c|c|c|c|c|c|}
\hline & \multicolumn{4}{|c|}{ PET Threshold Definition* } & \multicolumn{5}{|c|}{ Vd PET at $27 \%$ \& OP 1 Ref Vd T2 Object comparison } \\
\hline & $\begin{array}{l}\text { Object Vol of OP } 1 \\
\text { Ref T2 Object }\left(\mathrm{cm}^{3}\right)\end{array}$ & $\begin{array}{c}\text { Optimal PET } \\
\text { Threshold† (\%) }\end{array}$ & $\begin{array}{l}\text { Object Vol at Optimal } \\
\text { PET Threshold }\left(\mathrm{cm}^{3}\right)\end{array}$ & $\begin{array}{l}\text { Object Vol Vd PET } \\
\text { at } 27 \% \ddagger\left(\mathrm{cm}^{3}\right)\end{array}$ & $\begin{array}{l}\text { Intersecting } \\
\text { Vol }\left(\mathrm{cm}^{3}\right)\end{array}$ & $\begin{array}{l}\text { Union Vol } \\
\left(\mathrm{cm}^{3}\right)\end{array}$ & $\begin{array}{l}\text { Dice } \\
\text { Coeff }\end{array}$ & $\begin{array}{l}\mathrm{CD} \\
(\mathrm{mm})\end{array}$ & $\begin{array}{c}\text { Vol } \\
\text { Diff }\left(\mathrm{cm}^{3}\right)\end{array}$ \\
\hline \multicolumn{10}{|l|}{ Pt no. } \\
\hline 23 & 11.8 & 26 & 10.7 & 10.5 & 9.5 & 12.4 & 0.9 & 1.7 & 1.2 \\
\hline 25 & 12.7 & 27 & 12.9 & 12.9 & 10.0 & 15.0 & 0.8 & 2.7 & 0.2 \\
\hline 27 & 9.7 & 29 & 9.8 & 10.6 & 8.5 & 11.3 & 0.8 & 1.5 & 0.9 \\
\hline 31 & 9.8 & 26 & 9.9 & 9.6 & 8.3 & 10.7 & 0.9 & 1.4 & 0.2 \\
\hline Mean & 11.0 & 27 & 10.8 & 10.9 & 9.1 & 12.4 & 0.9 & 1.8 & 0.6 \\
\hline SD & 1.5 & 1.4 & 1.4 & 1.4 & 0.8 & 1.9 & 0.1 & 0.6 & 0.5 \\
\hline
\end{tabular}

Coeff = coefficient; Diff = difference; $O P$ = operator; pt = patient; ref = reference.

* To define a threshold for Vd PET, 4 patients with minimal pre-CED T2 signal were used to identify the threshold that best matched Vd PET and Vd T2.

$\dagger$ PET threshold resulting in best volumetric match between Vd PET and OP 1 reference T2 object.

$\ddagger 27 \%$ = mean optimal PET threshold.

generated at different PET thresholds. Table 1 shows each of the 4 patients with the PET threshold that best matched the volume of the reference object. The mean optimal threshold for these patients was $27 \% \pm 1.4 \%$. Dice coefficients of $0.9 \pm 0.1$ were measured between the volumes generated with a $27 \%$ threshold and the volumes of the reference objects (Table 1). This result led to the conclusion that a threshold of $27 \%$ was useful for autosegmentation of the distribution of 124I-omburtamab based on PET signal.

\section{Comparison of Vd PET at the $27 \%$ Threshold and Vd Sim}

Applying the $27 \%$ threshold, Vd PET volumes were calculated for all patients in the cohort. These volumes were then compared with the iPlan Flow simulated volumes for the 10 patients that met inclusion criteria (Fig. 5). Table 2 characterizes the similarity and spatial overlap of distribution determined based on iPlan Flow simulation and PET imaging using the $27 \%$ threshold. Eight of the 10 patients met all acceptance criteria. For 9 of 10 patients, more than $50 \%$ of the Vd Sim was found to be covered by the PET volume (PPV) and less than $50 \%$ of the PET volume was outside the simulated volume (FNR). For 2 patients, the Dice coefficient was less than 0.7 . These 2 patients were just outside the combined acceptance criteria. The Dice coefficient for both of these patients was 0.6, and the percentage of Vd PET that was outside Vd Sim (FNR) was not below $50 \%$ for 1 patient. The mean PPVs for these patients were still high, $70 \%$ and $80 \%$, respectively. Vd Sim corre-
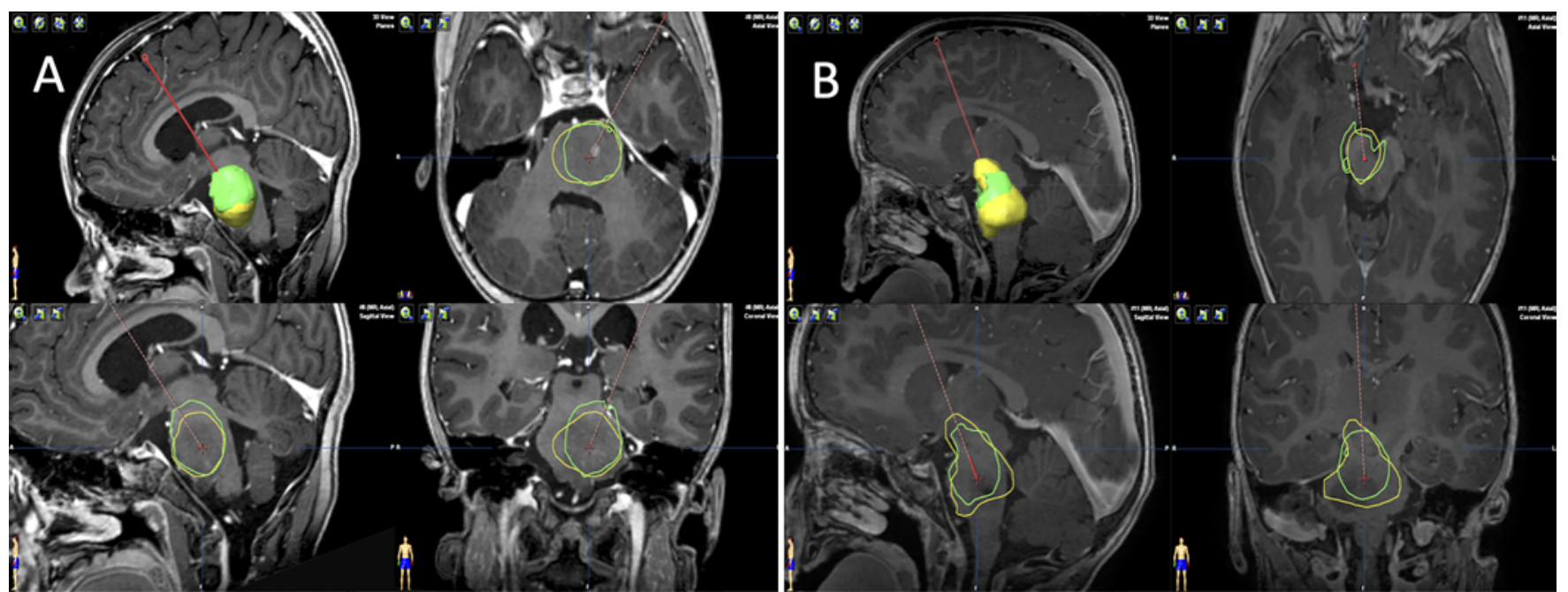

FIG. 5. Screenshot of the simulation software showing simulated volume (green) and Vd PET at 27\% (yellow) overlaid on 3-plane view 3D T1-weighted MR images in 2 representative patients. A: The combined acceptance criteria were met in this patient. Infusion of $3.84 \mathrm{ml}$ over a duration of 13 hours produced a Vd PET volume of $10.5 \mathrm{~cm}^{3}$, similar to the Vd Sim of $10.4 \mathrm{~cm}^{3} ; 76 \%$ of the simulated volume was found to be covered by the PET volume (PPV), and $25 \%$ of the PET volume was not part of the simulated volume (FNR). B: Infusion of $4.38 \mathrm{ml}$ into the brainstem over a duration of 8 hours produced a Vd PET at $27 \%$ volume of $16.2 \mathrm{~cm}^{3}$, whereas the Vd Sim was $9.0 \mathrm{~cm}^{3}$. The PPV was acceptable at 84\%; however, the FNR was $53 \%$ and therefore the combined acceptance criteria were not met in this patient. 
TABLE 2. Volume, similarity, and spatial overlap of Vd PET at $27 \%$ and Vd Sim

\begin{tabular}{|c|c|c|c|c|c|c|c|c|c|}
\hline & \multirow{2}{*}{$\begin{array}{c}\text { Vd PET } \\
27 \%\left(\mathrm{~cm}^{3}\right)\end{array}$} & \multirow{2}{*}{$\begin{array}{l}\text { Vd Sim } \\
\left(\mathrm{cm}^{3}\right)\end{array}$} & \multirow{2}{*}{$\begin{array}{l}\text { Intersecting } \\
\text { Vol }\left(\mathrm{cm}^{3}\right)\end{array}$} & \multirow{2}{*}{$\begin{array}{c}\mathrm{CD} \\
(\mathrm{mm})\end{array}$} & \multirow{2}{*}{$\begin{array}{c}\mathrm{AD} \\
(\mathrm{mm})\end{array}$} & \multirow{2}{*}{$\begin{array}{c}\text { Vol Diff } \\
\left(\mathrm{cm}^{3}\right)\end{array}$} & \multicolumn{3}{|c|}{ Acceptance criteria for similarity } \\
\hline & & & & & & & Dice Coeff & FNR (\%) & PPV (\%) \\
\hline \multicolumn{10}{|l|}{ Pt no. } \\
\hline 23 & 10.5 & 10.4 & 7.9 & 3.7 & 1.9 & 0.2 & 0.8 & 25 & 76 \\
\hline 24 & 13.9 & 10.9 & 7.6 & 4.3 & 3.4 & 3.0 & 0.6 & 45 & 70 \\
\hline 25 & 12.9 & 11.8 & 8.4 & 2.9 & 2.5 & 1.1 & 0.7 & 35 & 71 \\
\hline 26 & 11.0 & 10.8 & 7.9 & 3.6 & 2.2 & 0.2 & 0.7 & 29 & 73 \\
\hline 27 & 10.6 & 11.1 & 7.8 & 4.5 & 2.3 & 0.6 & 0.7 & 26 & 71 \\
\hline 30 & 14.5 & 9.0 & 8.1 & 2.2 & 2.3 & 5.5 & 0.7 & 44 & 90 \\
\hline 31 & 9.6 & 11.6 & 7.3 & 5.1 & 2.6 & 2.0 & 0.7 & 23 & 63 \\
\hline 32 & 11.6 & 10.7 & 8.2 & 4.1 & 2.1 & 0.9 & 0.7 & 30 & 76 \\
\hline 36 & 15.2 & 11.7 & 10.2 & 1.7 & 1.8 & 3.5 & 0.8 & 33 & 88 \\
\hline 37 & 16.2 & 9.0 & 7.5 & 6.4 & 3.2 & 7.2 & 0.6 & 53 & 84 \\
\hline Mean & 12.6 & 10.7 & 8.1 & 3.9 & 2.4 & 2.4 & 0.7 & 34 & 77 \\
\hline $\mathrm{SD}$ & 2.2 & 1.0 & 0.8 & 1.3 & 0.5 & 2.3 & 0.05 & 10 & 8 \\
\hline
\end{tabular}

lated with the geometry of Vd PET at $27 \%$, but the volume difference was too high and caused the mismatch. Overall, the mean PPV for all 10 patients was $77 \% \pm 8 \%$ and the mean FNR was $34 \% \pm 10 \%$. The mean Dice coefficient was $0.7 \pm 0.05$, the average $C D$ was $3.9 \pm 1.3 \mathrm{~mm}$, and $\mathrm{AD}$ was $2.4 \pm 0.5 \mathrm{~mm}$. These data show a strong overall agreement. Figure 5 shows two representative cases, one in which the patient met the combined acceptance criteria (Fig. 5A) and one in which the patient did not (Fig. 5B).

\section{Discussion}

In this study, the iPlan Flow infusion simulation algorithm was evaluated for use in the pediatric brainstem by comparing simulated outcomes with actual radiolabeled antibody infusions for the treatment of DIPG. The distribution of 124I-omburtamab as determined on PET images was compared to the distribution estimated by the simulation algorithm. Similarity metrics were used to quantify the similarity between the actual and the estimated infusate distribution. The combined acceptance criteria were met for 8 out of the 10 evaluated patients. In addition, the mean PPV (77\%) and FNR (34\%) were better than the values found in the previous validation of this algorithm for putamen and thalamus applications (73\% and $40 \%$, respectively). ${ }^{10}$ However, there were some deviations in volume and shape between Vd PET and Vd Sim that were likely due to the cumulative effect of multiple variables. The outcome of the interuser variability for T2 volumes demonstrated good overlap between users. Nevertheless, small differences could influence the accuracy of the defined PET threshold and therefore the reference standard. In addition, the method to define a PET percentage threshold has not been externally validated. Furthermore, resolution of PET imaging compared to MRI is relatively low and may lead to differences. It also remains unknown if predictive algorithms or actual distribution patterns may differ depending on differences in cellular affinity of therapeutic agents, even if infusion parameters remain constant. In this particular trial, the monoclonal antibody is a known bioactive substrate and the distribution of inert antineoplastics may differ. Even with all of these contributing factors, the simulation algorithm achieved strong overall agreement with actual distribution. For all patients, Vd Sim correlated with the geometry of Vd PET at 27\%. The mean distance between the centroids of the two objects, $3.9 \mathrm{~mm}$, was small. For context, the average longest diameter of the Vd PET object at $27 \%$ was $35.5 \mathrm{~mm}$, which is approximately 9 times higher than the average centroid shift between the $\mathrm{Vd}$ PET and Vd Sim objects. This gives a broad comparative measure of the objects and relative differences and shows a good match. This evaluation was based on one cohort within this study that had sufficient uniformity of infusion parameters in a large enough data set for analysis. While this is a limitation of how broadly this algorithm applies to different clinical settings, it should be noted that previous validations have been performed for different molecules and volumes/clinical parameters. ${ }^{9,10}$ Catheters in this study with their tips less than $0.5 \mathrm{~cm}$ from cystic/necrotic regions were excluded from analysis due to the likelihood of leakage and/or pooling (Fig. 3B). Catheter tip placement should be planned to be more than $0.5 \mathrm{~cm}$ from fluid-filled leakage pathways in order to avoid potential leakage and/ or pooling..$^{20}$ The software can provide a warning during the planning phase to minimize the risk of this complication (Fig. 3A and B).

The purpose for utilizing the software prospectively for planning CED within the brainstem is twofold: first, to warn of trajectories unlikely to provide intraparenchymal convection, due to leakage, and second, to estimate the likely fluid distribution and target coverage for acceptable trajectories. Segmentation of potential leakage pathways and 3D visualization of user-defined minimal distances from the catheter tip to a defined leakage pathway while planning support the user in identifying suitable trajectories. A warning will appear automatically if the catheter is too close to fluid-filled regions. For target coverage evaluation, a 1- or 2-cm margin around a predefined target object 
can be visualized and the predicted coverage information, based on the percentage of target volume that overlaps with the simulation result for a given trajectory, is calculated and shown. Given the demonstrated similarity between simulated and actual infusion volumes described in this study, testing a few different infusion scenarios allows the user to estimate and optimize target coverage prior to the procedure.

\section{Conclusions}

In conclusion, implemented guidelines in iPlan Flow aid to minimize the risk of infusate leakage and therefore support planning of trajectories that produce intraparenchymal convection. The simulation algorithm models the likely infusate distribution for a CED treatment in DIPG patients. The combination of trajectory planning guidelines and infusion simulation in the software can be used prospectively to enable optimization of personalized CED treatment.

\section{Acknowledgments}

We thank the patients and their families who participated in the trial. We also thank the following organizations and programs for research support: The Cure Starts Now; Solving Kids' Cancer; The Lyla Nsouli Foundation for Children's Brain Cancer Research; Cookies for Kids' Cancer; The Cristian Rivera Foundation; Battle for a Cure; Cole Foundation; Meryl \& Charles Witmer Charitable Foundation; Tuesdays with Mitch Charitable Foundation; The Ian's Friends Foundation; The Matthew Larson Foundation; The McKenna Claire Foundation; The Olivia Boccuzzi Foundation; The Children's Brain Tumor Family Foundation; The Pediatric Brain Tumor Foundation; The St. Baldrick's Foundation; Lyonhearted Foundation; ChadTough Foundation; Fly A Kite Foundation; Brooke Healey Foundation.

\section{References}

1. Warren KE. Beyond the blood:brain barrier: the importance of central nervous system (CNS) pharmacokinetics for the treatment of CNS tumors, including diffuse intrinsic pontine glioma. Front Oncol. 2018;8:239.

2. Bobo RH, Laske DW, Akbasak A, et al. Convection-enhanced delivery of macromolecules in the brain. Proc Natl Acad Sci U S A. 1994;91(6):2076-2080.

3. Song DK, Lonser RR. Convection-enhanced delivery for the treatment of pediatric neurologic disorders. J Child Neurol. 2008;23(10):1231-1237.

4. Shi M, Sanche L. Convection-enhanced delivery in malignant gliomas: a review of toxicity and efficacy. J Oncol. 2019; 2019:9342796.

5. Mehta AM, Sonabend AM, Bruce JN. Convection-enhanced delivery. Neurotherapeutics. 2017;14(2):358-371.

6. Chen MY, Lonser RR, Morrison PF, et al. Variables affecting convection-enhanced delivery to the striatum: a systematic examination of rate of infusion, cannula size, infusate concentration, and tissue-cannula sealing time. J Neurosurg. 1999;90(2):315-320.

7. Raghavan R, Brady ML, Rodríguez-Ponce MI, et al. Convection-enhanced delivery of therapeutics for brain disease, and its optimization. Neurosurg Focus. 2006;20(4):E12.

8. Raghavan R, Mikaelian S, Brady M, Chen ZJ. Fluid infusions from catheters into elastic tissue: I. Azimuthally symmetric backflow in homogeneous media. Phys Med Biol. 2010;55(1): 281-304.

9. Sampson JH, Raghavan R, Brady ML, et al. Clinical utility of a patient-specific algorithm for simulating intracerebral drug infusions. Neuro Oncol. 2007;9(3):343-353.

10. Rosenbluth KH, Eschermann JF, Mittermeyer G, et al. Analysis of a simulation algorithm for direct brain drug delivery. Neuroimage. 2012;59(3):2423-2429.

11. Zhou Z, Luther N, Ibrahim GM, et al. B7-H3, a potential therapeutic target, is expressed in diffuse intrinsic pontine glioma. J Neurooncol. 2013;111(3):257-264.

12. Maachani UB, Tosi U, Pisapia DJ, et al. B7-H3 as a prognostic biomarker and therapeutic target in pediatric central nervous system tumors. Transl Oncol. 2020;13(2):365-371.

13. Souweidane MM, Kramer K, Pandit-Taskar N, et al. Convection-enhanced delivery for diffuse intrinsic pontine glioma: a single-centre, dose-escalation, phase 1 trial. Lancet Oncol. 2018;19(8):1040-1050.

14. Sankey EW, Butler E, Sampson JH. Accuracy of novel computed tomography-guided frameless stereotactic drilling and catheter system in human cadavers. World Neurosurg. 2017; 106:757-763.

15. Richardson RM, Kells AP, Martin AJ, et al. Novel platform for MRI-guided convection-enhanced delivery of therapeutics: preclinical validation in nonhuman primate brain. Stereotact Funct Neurosurg. 2011;89(3):141-151.

16. Pandit-Taskar N, Kramer K, Humm J, et al. Imaging and quantitation with 124I-8H9 positron emission tomography (PET) in metastatic central nervous system (CNS) disease. $J$ Nucl Med. 2008;49(suppl 1):144P.

17. Besemer AE, Titz B, Grudzinski JJ, et al. Impact of PET and MRI threshold-based tumor volume segmentation on patient-specific targeted radionuclide therapy dosimetry using CLR1404. Phys Med Biol. 2017;62(15):6008-6025.

18. Richardson RM, Gimenez F, Salegio EA, et al. T2 imaging in monitoring of intraparenchymal real-time convectionenhanced delivery. Neurosurgery. 2011;69(1):154-163.

19. Sampson JH, Raghavan R, Provenzale JM, et al. Induction of hyperintense signal on T2-weighted MR images correlates with infusion distribution from intracerebral convectionenhanced delivery of a tumor-targeted cytotoxin. AJR Am J Roentgenol. 2007;188(3):703-709.

20. Sampson JH, Brady ML, Petry NA, et al. Intracerebral infusate distribution by convection-enhanced delivery in humans with malignant gliomas: descriptive effects of target anatomy and catheter positioning. Neurosurgery. 2007;60(2) (suppl 1):ONS89-ONS99.

21. Dice LR. Measures of the amount of ecologic association between species. Ecology. 1945;26(3):297-302.

22. Zhu M, Bzdusek K, Brink C, et al. Multi-institutional quantitative evaluation and clinical validation of Smart Probabilistic Image Contouring Engine (SPICE) autosegmentation of target structures and normal tissues on computer tomography images in the head and neck, thorax, liver, and male pelvis areas. Int J Radiat Oncol Biol Phys. 2013;87(4):809-816.

23. Taha AA, Hanbury A. Metrics for evaluating 3D medical image segmentation: analysis, selection, and tool. BMC Med Imaging. 2015; 15:29.

\section{Disclosures}

Eva Wembacher-Schroeder, Nicole Kerstein, and Rowena Thomson are employees of Brainlab. MSKCC has institutional financial interests related to this research in the form of intellectual property rights and equity interests in Y-mAbs, the company licensing the intellectual property from MSKCC. Y-mAbs has provided funding for this study.

\section{Author Contributions}

Conception and design: Wembacher-Schroeder, Kerstein, Thomson, Souweidane. Acquisition of data: Wembacher-Schroeder, Kerstein, Souweidane. Analysis and interpretation of data: 
Wembacher-Schroeder, Kerstein, Bander, Thomson, Souweidane. Drafting the article: Wembacher-Schroeder, Bander, Souweidane. Critically revising the article: Wembacher-Schroeder, Bander, Pandit-Taskar, Thomson, Souweidane. Reviewed submitted version of manuscript: all authors. Approved the final version of the manuscript on behalf of all authors: Wembacher-Schroeder. Statistical analysis: Kerstein, Bander, Thomson. Administrative/ technical/material support: Wembacher-Schroeder, Kerstein, Bander, Souweidane. Study supervision: Wembacher-Schroeder, Souweidane.

\section{Correspondence}

Eva Wembacher-Schroeder: Brainlab AG, Munich, Germany. eva.wembacher@brainlab.com. 